\title{
Early Postoperative Results of On-Pump Coronary Endarterectomy: Is Gender a Risk Factor?
}

\author{
Cüneyt Eriş, MD, Mesut Engin, MD, Sadık Ahmet Sunbul, MD, Ahmet Kağan, AS, MD, Burak Erdolu, MD \\ Department of Cardiovascular Surgery, University of Health Sciences, Bursa Yuksek Ihtisas Training and Research Hospital, \\ Yıldırım/Bursa, Turkey
}

\section{ABSTRACT}

Background: Coronary endarterectomy (CE) combined with coronary artery bypass grafting $(\mathrm{CABG})$ is an effective but still controversial surgical strategy for the treatment of diffuse coronary artery disease. In this study, we aimed to investigate the impact of gender differences on operative and early postoperative results of patients who underwent CABG with CE.

Methods: This retrospective study included 141 patients who had undergone CE combined with CABG from January 2015 to December 2020, as well as 141 patients without $\mathrm{CE}$ as the control group. First, patients with and without $\mathrm{CE}$ were compared. Next, patients undergoing $\mathrm{CE}$ were divided into 2 groups according to gender (group 1, male patients; group 2, female patients).

Results: Of the 141 patients who underwent CE combined with on-pump CABG, 95 (67.3\%) were male, and median age was 66 years (range 58 to 71.2). Of the 141 patients who underwent isolated on-pump CABG, 99 (70.2\%) were males, and median age was 63 years (range 41 to 80.4 ). The data for these 2 groups (with and without CE) were compared. Previous percutaneous coronary intervention (PCI), presence of diabetes mellitus, and perfusion time were significantly more common in the CE group. There were 95 patients in group 1 , with a median age of 65 years (range 58 to 69 ), and 46 patients in group 2, with a median age of 66 (64 to 71.2). There were no difference between the groups in terms of age, body mass index, hyperlipidemia, chronic obstructive pulmonary disease, peripheral artery disease, or previous coronary intervention. The need for positive inotropic support and postoperative atrial fibrillation were found to be significantly more common in group $2(\mathrm{P}=.022$ and .039 , respectively). Defibrillation after releasing the aortic cross clamp was also significantly more common in group $2(\mathrm{P}=.025)$.

Conclusion: In our study, the need for defibrillation after aortic cross-clamp releasing in the perioperative period, the need for inotropic support and the incidence of atrial

Received April 27, 2021; received in revised form fune 24, 2021; accepted fune 24, 2021.

Correspondence: Cüneyt Eriş, MD, Department of Cardiovascular Surgery, University of Health Sciences, Bursa Yuksek Ibtisas Training and Research Hospital, Emniyet St, Mimar Sinan Town, Yildrrm/BURSA, Turkey; 902242955000; fax: 902242756767 (e-mail:dr_ceris@hotmail.com). fibrillation in the post-operative period, increased significantly in the female gender. CE can be performed safely in both genders with acceptable mortality and morbidity rates.

\section{INTRODUCTION}

Recently, because of their reduced invasiveness and good results, percutaneous coronary interventions (PCIs) have become the treatment of choice for coronary artery disease (CAD). As a result, more complex patients with high comorbidity are now referred for CABG surgery [Eris 2021]. The principal aim in coronary artery bypass graft (CABG) surgery is to provide complete revascularization of diseased coronary arteries, because complete revascularization reduces the risk of perioperative myocardial infarction, in-hospital mortality, and relapse of late symptoms [Guvenc 2020].

Patients with diffuse calcific disease, long segment, or sequential coronary lesions constitute a controversial and challenging group for surgical revascularization. Therefore, an effective surgical strategy should be applied to patients with severe $\mathrm{CAD}$ to achieve complete revascularization. Coronary endarterectomy (CE) combined with CABG is a useful and sometimes necessary technique to provide complete revascularization [Russo 2017].

One unsettled issue is the effect of female gender on postoperative morbidity and mortality after CABG surgery. Women have less extensive obstructive disease but more diffuse coronary artery disease compared with men. They also have smaller coronary artery diameters than men; anastomosis to a narrower coronary artery is technically more difficult and may affect graft patency and clinical outcomes. Nevertheless, some studies have reported that women show better long-term survival than men [Toumpoulis 2006].

In this study, we aimed to determine whether gender differences affect early postoperative outcomes of on-pump $\mathrm{CABG}$ patients with adjunct $\mathrm{CE}$.

\section{METHODS}

This retrospective, single-center study was conducted from January 2015 to January 2020 at Bursa Yuksek Ihtisas Education and Research Hospital, Bursa, Turkey. We analyzed 1162 patients ( 800 men and 362 women), operated on by the same surgical team, who underwent on-pump CABG 
surgery during this time period. Of these patients, 141 (95 $(11,87 \%)$ men and $46(12,7 \%)$ women) had CE combined with on-pump CABG.. The patient group to whom CE was applied was divided into 2 groups of men (group 1) and women (group 2), and results were compared. In addition, 141 patients who underwent isolated CABG (without CE) were included according to a random numbers table from the full data set as the control group.

Approval of the Bursa Yuksek Ihtisas Education and Research Hospital Ethics Committee was obtained. The study was conducted in accordance with the Declaration of Helsinki Ethical Principles and Good Clinical Practices. Preoperative variables examined included age, gender, body mass index $[\mathrm{BMI}]$, diabetes mellitus, hypertension, smoking history, hypercholesterolemia, peripheral vascular disease, previous myocardial infarction (MI), preoperative left ventricular ejection fraction, intraoperative characteristics (cross-clamp times, perfusion times, number of bypass grafts performed, left internal mammary artery [LIMA] use, and defibrillation needs. Postoperative outcomes examined included MI, new atrial fibrillation, intra-aortic balloon pump (IABP), inotrope agents, low cardiac output, bleeding, hospital mortality, intensive care unit (ICU) stay times, and hospital discharge times. Patients who underwent on-pump CABG with no additional cardiac procedure were included in the study. We excluded patients with valvular heart disease, emergency cardiac surgical procedures, and concomitant surgeries. Data were obtained from our hospital's computerized database and retrospectively analyzed.

Blood samples were drawn from each patient 1 day before surgery and after 24 hours to measure the myocardial biomarker high-sensitivity troponin I (hsTnI). All patients had continuous electrocardiographic monitoring in the ICU. Twelve-lead electrocardiographic recordings were performed preoperatively, 1 hour postoperatively in the ICU, daily for at least the first 3 postoperative days, and at discharge. Left ventricular ejection fraction was measured with 2-dimensional echocardiography preoperatively and postoperatively before discharge.

\section{Surgical Technique}

All operations were performed under cardiopulmonary bypass with mild hypothermia $\left(30^{\circ}\right.$ to $\left.32^{\circ} \mathrm{C}\right)$ after cardioplegic arrest. During cardiopulmonary bypass, myocardial protection was provided in all cases, with antegrade and retrograde cold blood cardioplegia given every 20 minutes. Although the decision for applying CE was considered as an option during preoperative coronary angiographic examination, the actual decision and choice of technique were based on intraoperative findings of the target coronary arteries.

The basic principle of CE is complete removal of the plaque. Atherosclerotic plaque is removed from the proximal and distal parts of the coronary artery. To prevent competitive flow through the native coronary artery, we did not insist on complete removal of the plaque in the proximal part, but we paid special attention to provide the best possible flow in the distal part. Two main techniques, open and closed, can be used to perform the CE procedure. In all cases, the closed technique was our primary option, because it is quicker and simpler and requires a smaller anastomosis than open surgery. Open CE is performed when plaque extraction cannot be completed through a limited arteriotomy or when the plaque is broken, and sufficient exposure is needed to remove the atherosclerotic core completely. The distal arteriotomy incision is extended to the nondiseased segment. An example of the CE technique applied to a severely diseased vessel is shown in Figure 1.

\section{Definition of Postoperative Complications}

Perioperative myocardial infarction was defined as the formation of a new $Q$ wave in $\geq 2$ consecutive leads on the electrocardiogram and a $\mathrm{TnI}$ value $>10$ times the 99th percentile upper reference value within the first 48 hours after surgery. Postoperative respiratory failure was defined as the need for mechanical ventilation for $>48$ hours or a requirement for the presence of pneumonia. Postoperative cerebrovascular accident was defined as occurrence of a new stroke or intracranial hemorrhage confirmed by radiological imaging. New stroke that developed in the postoperative period in patients with preoperative stroke was defined as worsening of neurological deficit with new radiological findings. Postoperative infection was defined as pneumonia or septicemia requiring antibiotherapy and cases of mediastinitis involving deep mediastinal tissues causing sternal dehiscence, requiring reoperation. Bleeding was defined as tamponade causing hemodynamic instability and postoperative drainage requiring reoperation. Renal failure was defined as new need for dialysis after the operation. Operative death was defined as death occurring within 30 days after surgery, either in hospital or after discharge from the hospital. Atrial fibrillation was defined as the absence of a $\mathrm{P}$ wave before the QRS complex and the presence of an irregular rhythm in the postoperative term.

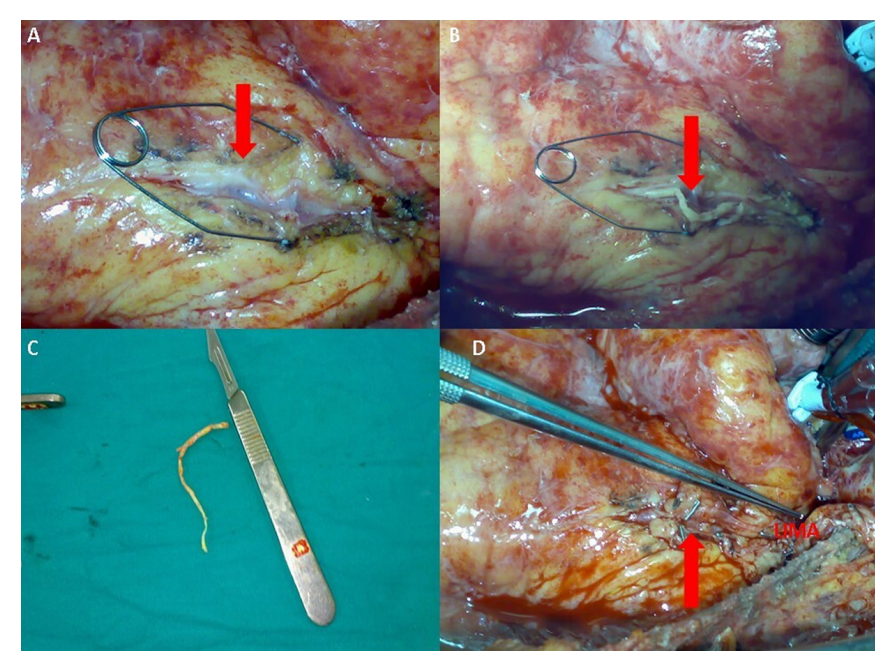

Figure 1. A, Diseased left anterior descending artery. B, Endarterectomy material after vascular arteriotomy. C, Completely removed endarterectomy material. D, Completed long segment LIMA to LAD artery anastomosis. 


\section{Statistical Analysis}

Statistical analyses were performed with Statistical Package for the Social Sciences version 23.0 (SPSS, Chicago, IL). Categorical variables are presented as number and percentage. Comparison of these variables were performed by $\chi^{2}$ test or Fisher's exact test. To show the distribution of continuous variables, the Kolmogorov-Smirnov normality test was used. For normally distributed continuous data, Student's t test was used to compare 2 independent groups, and variables are expressed as mean \pm standard deviation (SD). For

Table 1. Characteristics of Patients with and without Coronary Endarterectomy (CE)

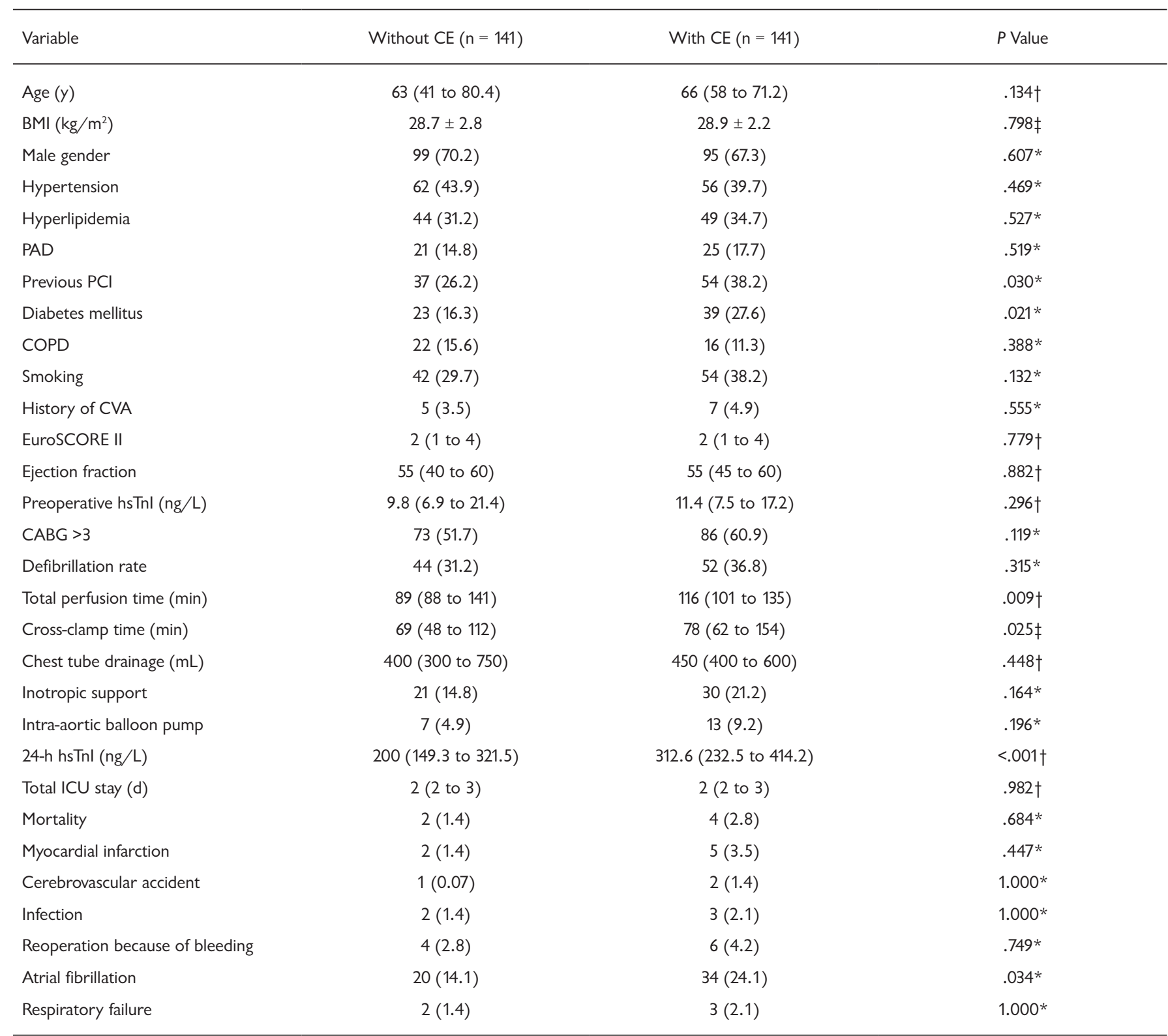

Data are median (interquartile range: 25 th to 75 th percentile), mean \pm SD, or $n(\%)$.

COPD indicates chronic obstructive pulmonary disease; CVA, cerebrovascular accident; PAD, peripheral artery disease; EuroSCORE II, European System for Cardiac Operative Risk Evaluation II.

* $\chi^{2}$ test (Fisher's exact test for values $<5$ ).

$\dagger$ †ann-Whitney $U$ test.

$\ddagger$ Student's $t$ test. 
non-normally distributed continuous data, Mann-Whitney $U$ test was used to compare 2 independent groups, and variables are expressed as median (25th to 75 th percentile). $P$ value $<.05$ was accepted as statistically significant for all comparisons.

\section{RESULTS}

Of the 141 patients who underwent CE combined with on-pump CABG, 95 (67.3\%) were males, with a median age of 66 years (range 58 to 71.2 ). Of the 141 patients who underwent isolated on-pump CABG, 99 (70.2\%) were males, with a median age of 63 (range 41 to 80.4). The data for these 2 groups (with and without CE) were compared. Previous PCI, diabetes mellitus, and postoperative atrial fibrillation were more common in the CE group, and perfusion times were significantly higher. Other patient characteristics and postoperative complication rates were similar between the 2 groups (Table 1).

Demographic data of patients with CE are given in Table 2. Group 1 contained 95 male patients with a median age of 65 years (range 58 to 69); group 2 contained 46 female patients with a median age of 66 (range 64 to 71.2). There were no differences between the groups in terms of age, body mass index, hyperlipidemia, chronic obstructive pulmonary disease, peripheral artery disease, or previous coronary intervention.
Hypertension and diabetes mellitus were found to be significantly more common in group $2(P=.005, P=.012$, respectively), and smoking was significantly more common in group $1(P=.038)$.

Operative data of the patients are given in Table 2. There were no differences between the groups in the number of bypassed vessels, the number of vessels on which $\mathrm{CE}$ was performed, use of the left internal mammary artery, or perfusion times. Defibrillation after releasing the aortic cross clamp was significantly more common in group $2(P=.025)$.

Postoperative characteristics and complication rates of the patient groups are given in Table 3. There were no statistically significant differences between the 2 groups in terms of duration of hospital and intensive care stays, mortality, postoperative MI, cerebrovascular events, infection, chest tube drainage amounts, reoperation due to bleeding, respiratory failure, or renal failure. Positive inotropic support and postoperative atrial fibrillation were found to be significantly more common in group $2(P=.022$ and .039 , respectively).

\section{DISCUSSION}

Although CE is a complex procedure, it is an essential and sometimes necessary method to achieve complete revascularization in patients with diffuse CAD. CE can be performed safely with acceptable operative and early postoperative

Table 2. Demographic Data and Preoperative Features of Patients with CE

\begin{tabular}{lccl}
\hline Variable & Men (Group 1) $(\mathrm{n}=95)$ & Women (Group 2) (n= 46) & P Value \\
\hline Age $(\mathrm{y})$ & $65(58$ to 69$)$ & $66(64$ to 71.2$)$ & $.118 \dagger$ \\
BMI $\left(\mathrm{kg} / \mathrm{m}^{2}\right)$ & $28.6 \pm 1.7$ & $29.2 \pm 2.8$ & $.154 \ddagger$ \\
Hypertension & $30(31.6)$ & $26(56.5)$ & $.005^{*}$ \\
Hyperlipidemia & $32(33.7)$ & $17(37)$ & $.702 *$ \\
PAD & $19(20)$ & $19(41.3)$ & $.311 *$ \\
Previous PCl & $35(36.8)$ & $19(41.3)$ & $.609 *$ \\
Diabetes mellitus & $20(21)$ & $4(8.6)$ & $.012 *$ \\
COPD & $12(12.6)$ & $12(26)$ & $.581 *$ \\
Smoking & $42(44.2)$ & $3(6.5)$ & $.038 *$ \\
History of CVA & $4(4.2)$ & $2(1$ to 4$)$ & $.683 *$ \\
EuroSCORE II & $2(1$ to 3$)$ & 50 (45 to 60$)$ & $.525 \dagger$ \\
Ejection fraction & $55(45$ to 60$)$ & $11.4(7.5$ to 14$)$ & $.986 \dagger$ \\
hsTnl (ng/L) & $11.4(7.7$ to 17.2$)$ & $.371 \dagger$ \\
\hline
\end{tabular}

Data are median (interquartile range: 25 th to 75 th percentile), mean $\pm \mathrm{SD}$, or $\mathrm{n}(\%)$.

COPD indicates chronic obstructive pulmonary disease; CVA, cerebrovascular accident; PAD, peripheral artery disease; EuroSCORE II, European System for Cardiac Operative Risk Evaluation II.

* $\chi^{2}$ test (Fisher's exact test for values $<5$ ).

†Mann-Whitney $U$ test.

$\ddagger$ Student’s $t$ test. 
outcomes. In terms of postoperative complications, only the rate of postoperative atrial fibrillation was significantly higher in patients with CE in our study. Despite initial negative results in the past decades [Livesay 1986], several recent publications have shown that $\mathrm{CE}$ combined with CABG is a useful surgical option that has been accepted with its early and long-term outcomes in diffuse and calcific CAD [Russo 2017].

Cardiovascular disease is one of the most important causes of death in both male and female populations [Walli-Attaei 2020]. Therefore, the incidence of interventional coronary procedures and CABG is increasing all over the world. Today, as a result of the widespread implementation of PCIs, the patient population referred for CABG includes cases that are increasingly complex. In the current study, we demonstrate that $\mathrm{CE}$ with on-pump CABG can be performed in both genders with similar early postoperative complication rates. We found that postoperative positive inotropic support and postoperative atrial fibrillation were statistically more common in the female patient group compared with male patients.

A principal goal of CABG is to achieve complete revascularization, mainly of the left anterior descending artery (LAD), because incomplete revascularization of the coronary arteries has been proven to be a predictor of poor outcomes and high mortality rates after CABG [Tiruvoipati 2005a; Cameron 1996]. Up to $25 \%$ of patients with extensive CAD cannot be safely and successfully treated with standard CABG. Therefore, the technique of $\mathrm{CE}$, although sometimes risky, may become mandatory [Santini 2002]. The application of concomitant CE with CABG is $0.8 \%$ to $25 \%$ in the literature [Ghatanatti 2017]. Concomitant CE was 12.1\% (141/1162) in our patient group.

Many studies have shown that female gender is an indicator of poor outcomes and high mortality rates after CABG [Ranucci 2008]. It was suggested that these poor results may be due to the more numerous comorbid factors such as diabetes mellitus and hypertension in female patients [Goksedef 2012]. Additionally, some anatomic and biological mechanisms have been suggested to explain these poor postoperative surgical outcomes in women. Differences in outcomes between genders may be attributed to several factors: women have small body size, women's coronary arteries are thinner than those of men, and women have less coronary collateral flow than men [Kim 2007; Sheifer 200]. In our study, as in other studies [Alam 2013], we found that the prevalence of hypertension was higher in female patients compared with men, and smoking was more common in men. In addition, we found that the rate of diabetes mellitus was higher in the female patient

Table 3. Operative Variables of Patients with CE

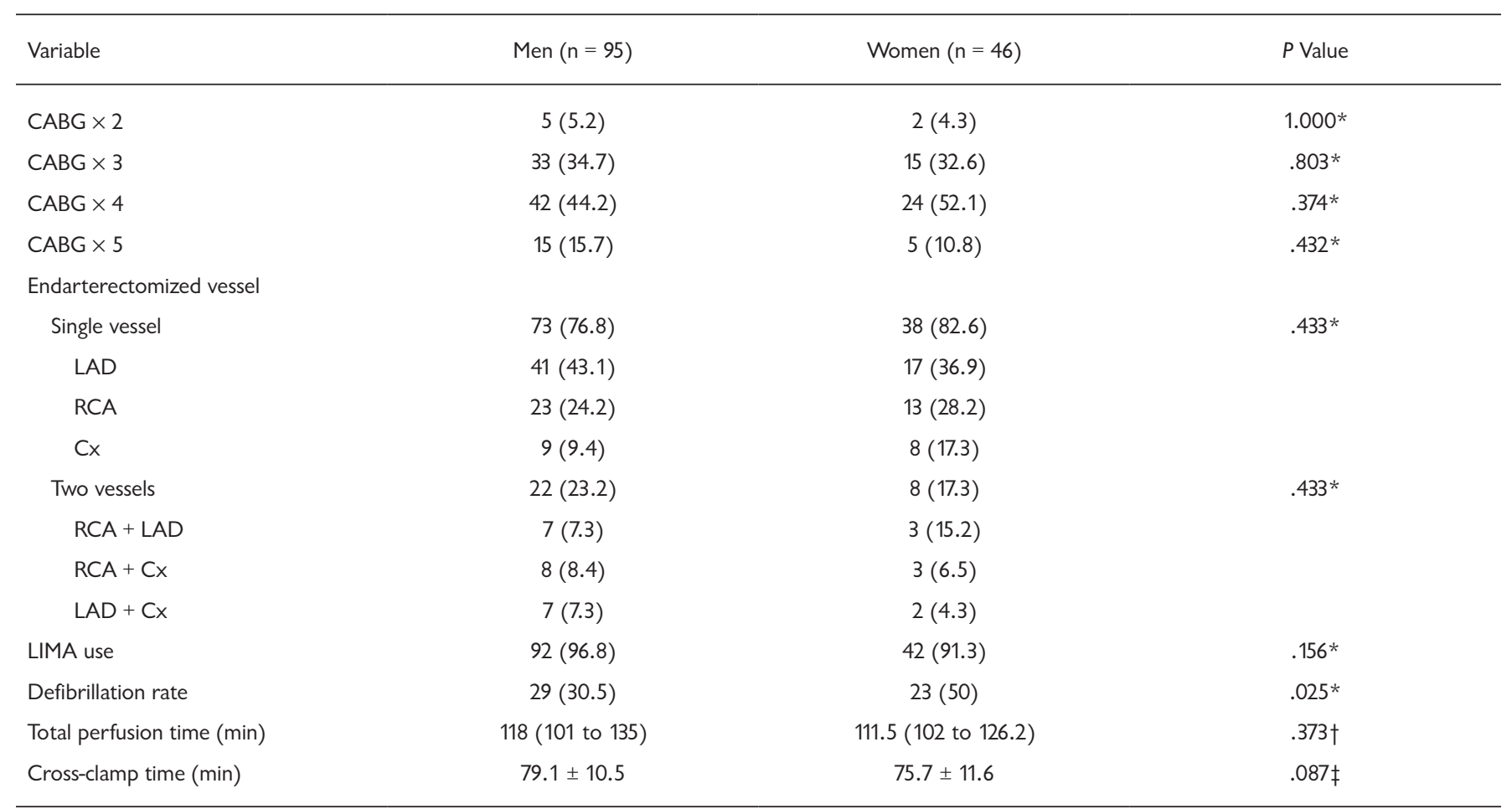

Data are $\mathrm{n}(\%)$, median (interquartile range: 25 th to 75 th percentile), or mean \pm SD.

Cx indicates circumflex artery; RCA, right coronary artery.

* $\chi^{2}$ test (Fisher's exact test for values $<5$ ).

†Mann-Whitney $U$ test.

$\ddagger$ Student’s $t$ test. 
group. In the current study, in the light of this information, the rates of atrial fibrillation and need for inotropic support were significantly higher in the female patient group.

However, in a recent study, early period results rates after CABG operations in patients $>70$ years old were found to be similar in female and male patients [Yuksel 2017]. Various studies in the literature have investigated the effect of gender on clinical outcomes after cardiovascular interventions. In the study conducted by Sannino et al [2018], the effect of gender on clinical outcomes after transcatheter aortic valve implantation was investigated. The authors found that early vascular complications and severe bleeding rates were higher in female patients. However, 1-year mortality rates were found to be lower in female patients [Sannino 2018]. In a study conducted by Trabattoni et al [2019], the results of left main coronary stenting were compared in terms of gender. At the end of the study, female gender was determined as an independent predictor for major adverse cardiovascular events [Trabattoni 2019]. Also, in a recent study, clinical results after off-pump CABG operations with CE were compared in terms of gender. Although there was no statistically significant difference between the groups in terms of early and long-term clinical outcomes, it was concluded that female gender had a higher risk [Sabzi 2020].

Aldea et al [1999] reported that the number of endarterectomized vessels was higher in men. In addition, it had been reported that 3-vessel disease has a high prevalence in men [Abramov 2000]. In contrast, Amato et al [2004] reported similar rates of $\mathrm{CE}$ in both genders. No significant difference was observed between male and female patients in terms of the number of diseased vessels and the number of bypasses performed in on-pump CABG patients with CE in our study. Additionally, the rate of CE was higher in males.

Perioperative $\mathrm{MI}$ is an important issue and may be the one of the reasons for increased mortality of CE. The perioperative myocardial infarction rate ranges from $3 \%$ to $25 \%$ in the literature [Stavrou 2016]. This rate was found to be $6.5 \%$ in female patients and $2.1 \%$ in male patients in our study $(\mathrm{P}=.330)$.

The basic principle of $\mathrm{CE}$ is the complete removal of the atherosclerotic core without leaving an obstructing plaque. In the closed technique, a shorter arteriotomy and thus a shorter anastomosis is performed, but it requires more experience. Distal and proximal embolism risk is higher, since most of the procedure is performed in a closed area. On the other hand, the open method is safer in terms of distal embolism, since complete cleaning of the atherosclerotic core is performed under direct vision. Because the arteriotomy is longer, it requires a longer anastomosis and therefore a longer ischemic time [Tiruvoipati 2005b; Takahashi 2013]. We used open endarterectomy and long anastomosis of LIMA as an onlay patch for reconstruction of the endarterectomized LAD in all cases. In our study group, although the rate of perioperative MI was lower than the literature, no statistically significant difference was found between men and women undergoing $\mathrm{CABG}$ and $\mathrm{CE}$.

Costa et al [2020] stated that LAD is the most common endarterectomized vessel that feeds a significant part of the left

Table 4. Postoperative features and complications of the patients with CE

\begin{tabular}{|c|c|c|c|}
\hline Variable & Men $(n=95)$ & Women $(n=46)$ & $P$ Value \\
\hline Chest tube drainage $(\mathrm{mL})$ & 450 (400 to 550$)$ & 500 (400 to 600$)$ & $.178 \dagger$ \\
\hline Inotropic support & $15(15.8)$ & $15(32.6)$ & $.022 *$ \\
\hline Intra-aortic balloon pump & $7(7.4)$ & $6(13)$ & $.275^{*}$ \\
\hline 24-h hsTnl (ng/L) & $303.2(232.5$ to 414.2$)$ & 337.6 (291.8 to 376.6$)$ & $.093 \dagger$ \\
\hline Total ICU stay $(\mathrm{d})$ & $2(2$ to 3$)$ & $2(2$ to 3$)$ & $.871 \dagger$ \\
\hline Total hospital stay (d) & $7(7$ to 8$)$ & 7 (7 to 9) & $.317 \dagger$ \\
\hline Ejection fraction & 50 (40 to 55$)$ & 45 (40 to 50$)$ & $.257 \dagger$ \\
\hline Mortality & $2(2.1)$ & $2(4.3)$ & $.596 *$ \\
\hline Myocardial infarction & $2(2.1)$ & $3(6.5)$ & $.330 *$ \\
\hline Cerebrovascular accident & $1(1.1)$ & $1(2.2)$ & $.548 *$ \\
\hline Infection & $1(1.1)$ & $2(4.3)$ & $.248 *$ \\
\hline Reoperation due to bleeding & $2(2.1)$ & $4(8.6)$ & $.089 *$ \\
\hline Atrial fibrillation & $18(18.9)$ & $16(34.8)$ & $.039 *$ \\
\hline Respiratory failure & $2(2.1)$ & $1(2.2)$ & $1.000 *$ \\
\hline Renal failure & $2(2.1)$ & $3(6.5)$ & $.330 *$ \\
\hline
\end{tabular}

Data are median (interquartile range: 25 th to 75 th percentile) or $n(\%)$.

* $\chi^{2}$ test (Fisher's exact test for values $<5$ ).

†Mann-Whitney $U$ test. 
heart and also is the most important target artery for complete revascularization. In our study, $54.6 \%$ of the patients underwent LAD endarterectomy. We observed that RCA was the second most common endarterectomized vessel at $40.4 \%$. This ratio varies between $21.1 \%$ and $83 \%$ in the literature [Stavrou 2016].

Reoperation due to postoperative bleeding was higher in the female group (2.1\% versus $8.6 \%$ ), but the difference was not significant. In addition, postoperative atrial fibrillation and postoperative inotropic need rates were significantly higher in the female group. These differences in outcomes may be attributed to several factors, such as women having smaller vessel size and less collateral flow than men [Goksedef 2012].

\section{Limitations}

Our study had several limitations. First, it was a singlecenter, retrospective study. The number of patients was relatively small in both groups. Also, our study focused on clinical follow-up, and graft patency was not examined with imaging techniques (i.e., conventional or computed tomographic angiography). Therefore, further studies with a larger number of patients, longer follow-up, and patency evaluation with angiographic results will be necessary to precisely define the true benefit of $\mathrm{CE}$ in addition to CABG.

\section{Conclusions}

In the presence of diffuse and calcific coronary artery disease, or when diffuse atheromatous plaques affect major lateral branches, CE may be the only and the mandatory surgical option when conventional grafting is impossible to provide complete revascularization. In our study, the need for defibrillation after aortic cross-clamp releasing in the perioperative period, the need for inotropic support and the incidence of atrial fibrillation in the post-operative period, increased significantly in the female gender. Although female gender has some disadvantages, CE can be performed safely in both genders with acceptable mortality and morbidity, as a result of the increased surgical experience and advances in myocardial protection techniques.

\section{REFERENCES}

Abramov D, Tamariz MG, Sever JY, et al. The influence of gender on the outcome of coronary artery bypass surgery. Ann Thorac Surg 2000;70:800-805.

Alam M, Bandeali SJ, Kayani WT, et al. Comparison by meta analysis of mortality after isolated coronary artery bypass grafting in women versus men. Am J Cardiol 2013;112:309-317.

Aldea GS, Gaudiani JM, Shapira OM, et al. Effect of gender on postoperative outcomes and hospital stays after coronary artery bypass grafting. Ann Thorac Surg 1999;67:1097-1103.

Amato VL, Timerman A, Paes AT, et al. Immediate results of myocardial revascularization. Comparison between men and women [in Portuguese]. Arq Bras Cardiol 2004.;3(Spec No):14-20.

Cameron A, Davis KB, Green G, Schaff HV. Coronary bypass surgery with internal-thoracic-artery grafts-effects on survival over a 15 -year period. N Engl J Med 1996;334:216-219.
Costa MACD, Betero AL, Okamoto J, et al. Coronary endarterectomy: a case control study and evaluation of early patency rate of endarterectomized arteries. Braz J Cardiovasc Surg 2020;35:9-15.

Eris C, Erdolu B, Engin M, et al. The effects of aortic clamping strategy on myocardial protection and early postoperative outcomes during coronary artery bypass grafting operations. Heart Surg Forum 2021;24:E217-E222.

Ghatanatti R, Teli A. Coronary endarterectomy: recent trends. J Clin Diagn Res 2017;11:PE01-PE04.

Göksedef D, Ömeroğlu SN, Balkanay OO, et al. Coronary artery bypass in women: what is really different? Turk Gogus Kalp Dama 2012;20:8-13.

Güvenç O, Göncü MT, Engin M, et al. Effects of coronary endarterectomy on postoperative early results in long segment coronary artery disease. Eur Res J 2020;6:187-192.

Kim C, Redberg RF, Pavlic T, Eagle KA. A systematic review of gender differences in mortality after coronary artery bypass graft surgery and percutaneous coronary interventions. Clin Cardiol 2007;30:491-495.

Livesay JJ, Cooley DA, Hallman GL, et al. Early and late results of coronary endarterectomy: analysis of 3,369 patients. J Thorac Cardiovasc Surg 1986;92:649-660.

Russo M, Nardi P, Saitto G, et al. Single versus double antiplatelet therapy in patients undergoing coronary artery bypass grafting with coronary endarterectomy: mid-term results and clinical implications. Interact Cardiovasc Thorac Surg 2017;24:203-208.

Santini F, Casali G, Lusini M, et al. Mid-term results after extensive vein patch reconstruction and internal mammary grafting of the diffusely diseased left anterior descending coronary artery. Eur J Cardiothorac Surg 2002;21:1020-1025.

Ranucci M, Pazzaglia A, Bianchini C, et al. Body size, gender, and transfusions as determinants of outcome after coronary operations. Ann Thorac Surg 2008;85:481-486.

Sabzi F, Asadmobini A. Gender difference in long- and short-term outcomes of off-pump coronary endarterectomy. Ann Card Anaesth 2020;23:419-424.

Sannino A, Szerlip M, Harrington K, et al. Comparison of baseline characteristics and outcomes in men versus women with aortic stenosis undergoing transcatheter aortic valve implantation. Am J Cardiol 2018;121:844-849.

Sheifer SE, Canos MR, Weinfurt KP, et al. Sex differences in coronary artery size assessed by intravascular ultrasound. Am Heart J 2000;139:649-653.

Stavrou A, Gkiousias V, Kyprianou K, et al. Coronary endarterectomy: the current state of knowledge. Atherosclerosis 2016;249:88-98.

Takahashi M, Gohil S, Tong B, et al. Early and mid-term results of offpump endarterectomy of the left anterior descending artery. Interact Cardiovasc Thorac Surg 2013;16:301-305.

Tiruvoipati R, Loubani M, Lencioni M, et al. Coronary endarterectomy: impact on morbidity and mortality when combined with coronary artery bypass surgery. Ann Thorac Surg 2005;79:1999-2003.

Tiruvoipati R, Loubani M, Peek G. Coronary endarterectomy in the current era. Curr Opin Cardiol 2005;20:517-520.

Toumpoulis IK, Anagnostopoulos CE, Balaram SK, et al. Assessment of independent predictors for long-term mortality between women and men after coronary artery bypass grafting: are women different from men? J Thorac Cardiovasc Surg 2006;131:343-351. 
Trabattoni D, Teruzzi G, Montorsi P, et al. Comparison of the effectiveness of percutaneous intervention of the left-main coronary artery with everolimus-eluting stents in women -vs- men. Am J Cardiol 2019;124:1357-1362.

Walli-Attaei M, Joseph P, Rosengren A, et al. Variations between women and men in risk factors, treatments, cardiovascular disease incidence, and death in 27 high-income, middle-income, and low-income countries (PURE): a prospective cohort study. Lancet 2020;396:97-109.

Yüksel A, Kan II, Yolgösteren A, et al. Are the early postoperative outcomes of coronary artery bypass grafting surgery in elderly women worse compared to men's? Braz J Cardiovasc Surg 2017;32:191-196. 